\title{
Electrodynamic Tether at Jupiter-I: Capture Operation and Constraints
}

\author{
Juan R. Sanmartin, Mario Charro, Enrico C. Lorenzini, Henry B. Garrett, \\ Claudio Bombardelli, and Cristina Bramanti
}

\begin{abstract}
Tethered spacecraft missions to the Jovian system suit the use of electrodynamic tethers because: 1) magnetic stresses are 100 times greater than at the Earth; 2) the stationary orbit is one-third the relative distance for Earth; and 3) moon $I o$ is a nearby giant plasma source. The (bare) tether is a reinforced aluminum foil with tens of kilometer length $L$ and a fraction of millimeter thickness $h$, which collects electrons as an efficient Langmuir probe and can tap Jupiter's rotational energy for both propulsion and power. In this paper, the critical capture operation is explicitly formulated in terms of orbit geometry and established magnetic and thermal plasma models. The design parameters $L$ and $h$ and capture perijove radius $r_{p}$ face opposite criteria independent of tape width. Efficient capture requires a low $r_{p}$ and a high $L^{3 / 2} / h$ ratio. However, combined bounds on tether bowing and tether tensile stress, arising from a spin made necessary by the low Jovian gravity gradient, require a high $r_{p}$ and a low $L^{5 / 2} / h$ ratio. Bounds on tether temperature again require a high $r_{p}$ and a low $L^{3 / 8} /(\text { tether emissivity })^{1 / 4}$ ratio. Optimal design values are discussed.
\end{abstract}

\section{INTRODUCTION}

A FULL study of the giant complex Jovian system is a central goal in planetary science. Missions of interest include getting a spacecraft (SC) into low orbit around moons Europa and Io and Jupiter itself. Basic issues made manifest in the Galileo mission involve power and propulsion needs, trip times, and harsh radiation environment. A succession of Jovian mission proposals have followed each other: Europa Orbiter, Jovian Icy Moons Orbiter, Europa Geophysical Explorer, Io Jovicentric Orbiter, Ganymede Exploration Orbiter, and Jovian Minisat Explorer [1]-[6]. Proposals moved from radioisotope thermal generators to nuclear reactors and solar arrays, for power, and from rockets to electrical thrusters with a variety of powering sources, for propulsion. Gravity assists were con- sidered for moon tours, while direct rather than gravity-assisted trips to Jupiter (as in the protracted Galileo mission) were also considered. Whereas Galileo was designed for $0.15-\mathrm{Mrad} S i$ radiation dose and accumulated 0.7-Mrad $\mathrm{Si}$ at its extended mission end, 3-Mrad $S i$ dose values are now being contemplated. NASA's mission presently prepared for $2011, J U N O$, will have a polar orbit to avoid radiation belts and allow in situ polar cap studies; it will have no access to Europa, however, and will make a somewhat problematic use of solar cells for power. A joint NASA/ESA flagship mission is, at present, actively planned for launch by 2016 to set an orbiter around Europa and additional 1-2 orbiters at Jupiter itself [7]-[12].

The use of electrodynamic (ED) tethers in Jupiter was previously considered by several authors. Penzo [13] and Hammond et al. [14] took cursory looks at potential applications at Jupiter. Gabriel et al. considered a number of operations using light-ion emitters for anodic contact, thus avoiding dependence on the ambient plasma [15]. Gallagher et al. used an ad hoc model of an anodic collection of electrons and a model of the $I o$ torus plasma density, which does not extend to the plasmasphere [16], [17]. Several ED-tether missions in Low Earth Orbit, TSS 1 [18], PMG [19], and TSS 1R [20]-[23] were carried out in the last decade. The first ED bare-tether mission, ProSEDS, ready for launch on March 29, 2003, was cancelled by NASA, following the Columbia accident two months before [24].

The approach discussed in this paper involves an ED bare tether, which, in principle, could tap Jupiter's rotational energy for both power and propulsion. The positions of perijove and apojove in equatorial elliptical orbits relative to the stationary circular orbit, at a radius $a_{s}$, which lies at an energy maximum in the orbit/planet-spin interaction, could be exploited to conveniently make the induced Lorentz force to be drag or thrust while generating power and navigating the system. A tether is just a mechanism to dissipate energy.

The nonrelativistic transformation of electric field reads

$\bar{E}($ tether frame $)-\bar{E}$ (plasma frame)

$$
\equiv \bar{E}_{m}=\left(\bar{v}-\bar{v}_{\mathrm{pl}}\right) \times \bar{B}
$$

where $\bar{v}$ and $\bar{v}_{\mathrm{pl}}$ are the velocities of SC and local corotating plasma, respectively, and $\bar{B}$ is the Jovian magnetic field. With the electric field outside the tether negligible in the frame moving with the plasma, there is an outside field $\bar{E}_{m}$ in the tether frame that will drive a current $\bar{I}$ inside the tether with $\bar{I} \cdot \bar{E}_{m}>0$. Using the Lorentz force $L \bar{I} \times \bar{B}$ on a tether of length $L$, Newton's third law for magnetic forces between 
steady-current systems proves that there is a net power loss in the tether-plasma interaction

$$
L \bar{I} \times \bar{B} \cdot \bar{v}+(-L \bar{I} \times \bar{B}) \cdot \bar{v}_{\mathrm{pl}}=-\bar{I} \cdot \bar{E}_{m} L<0
$$

which is power naturally feeding the tether electric circuit. The Lorentz force would be thrust if $\bar{v}$ is opposite the SC velocity relative to the plasma $\bar{v}-\bar{v}_{\mathrm{pl}}$, which is the case for prograde circular equatorial orbits at radius $a>a_{s}$ [25].

ED-tether operation requires effective electric contact with the ambient plasma. State-of-the-art hollow cathodes are very effective in establishing cathodic contact. The problem of anodic contact with a highly rarefied plasma was solved in 1992, when it was proposed that, instead of using a big end collector, the tether be left bare of insulation to allow it to collect electrons over the segment, resulting positively polarized, as a giant cylindrical Langmuir probe in the orbital-motionlimited (OML) regime [26]. Collection is efficient if the crosssectional dimension is thin, the collecting area still being large because the anodic segment may be tens of kilometers long. A thin foil collects the same OML current as a round wire of equal cross-sectional perimeter and will be much lighter [27]; the optimal tether thus presents three disparate dimensions, $L \gg w$ (foil width) $\gg h$ (foil thickness).

The Jovian system is particularly appropriate for thrusting as well as dragging by an ED tether with no external power. Operation requires plasma beyond the radius $a_{s}$ to be 1) dense enough and 2) corotating with the planet. Jupiter has both low mean density $\rho_{J}$ and rapid spin $\Omega_{J}$; as a result, its stationary orbit lies at

$$
a_{s}=\left(\mu_{J} / \Omega_{j}^{2}\right)^{1 / 3} \propto R_{J}\left(\rho_{J} / \Omega_{j}^{2}\right)^{1 / 3} \Rightarrow a_{s} \approx 2.24 R_{J}
$$

( $\mu_{J}$ and $R_{J}$ being Jupiter's gravitational parameter and radius, respectively) which is one-third the relative distance for the Earth. Furthermore, magnetic stresses at the surface are 100 times greater at Jupiter than at the Earth, the Jovian plasmasphere reaching to about $3.8 R_{J}$. Also, moon $I o$ is at 1:2 Laplace resonance with Europa and ten times relatively closer to its planet than the Moon is to the Earth. Extreme tectonics and volcanism from tidal deformations inside $I o$ make it continuously eject gas that is ionized and accelerated in the magnetosphere and made to corotate as a giant dense plasma torus, which would allow thrusting beyond the plasmasphere.

In the tether scheme, drag/thrust would only be effective within either plasmasphere or torus, with tether current (nearly) shut off at convenience by switching off hollow cathodes or plugging a large resistance in the tether circuit. Also, the tether could serve as power source whenever an electric load is plugged in. A large energy could be tapped (used locally or saved for later use) from the high power developed during SC capture and other high-current operations, with negligible effect on its dynamics; current could also be switched on away from such operations to just generate power.

In a mission scheme, the apojove could be lowered down to the orbits of Galilean moons through a sequence of perijove passes following capture, allowing frequent moon flybys, or lowered all the way to reach a low circular orbit around Jupiter [Fig. 1(a)]. Also, with the apojove down in the fast-flowing

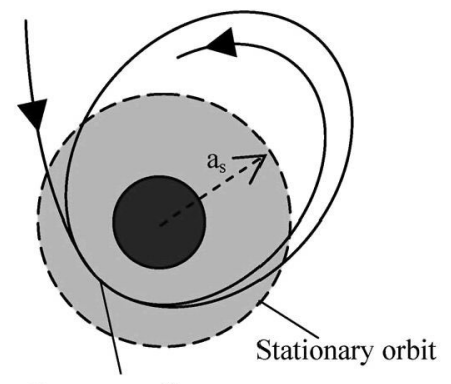

Drag at perijove

(a)

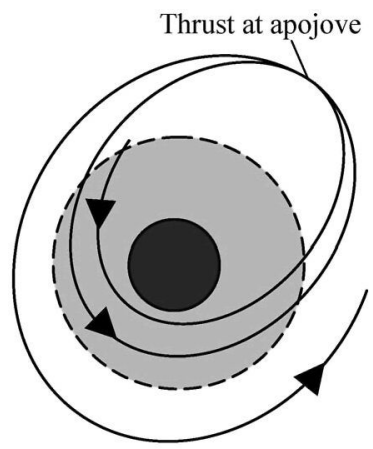

(b)
Fig. 1. Jovian tour phases. (a) Capture and lowering apojove. (b) Raising perijove.

plasma torus, switching the tether current off around perijove and on around apojove would produce a sequence of orbits with increasingly higher perijove to finally carry the SC deep inside the torus, allowing, in principle, insertion to $I o$ orbit [Fig. 1(b)] [25].

This paper deals with the Jupiter capture operation and associated constraints; the analysis of missions following capture will be presented in a companion paper [28]. Section II gives explicit formulas for the capture operation, in terms of accurate orbit geometry and Jovian magnetic model, for an arbitrary current collection law. The actual bare-tether current, depending on a standard thermal plasma model, is determined in Section III. Mission design constraints arising from heating effects and from bowing versus tensile stress effects are analyzed in Sections IV and V, respectively. Conclusions are discussed in Section VI.

\section{SC CAPTURE OpERATION}

SC capture is particularly critical for a tether as compared with a propellant-consuming thruster, which faces a separate issue in attaining closed-orbit evolution, whereas closed orbits, in the tether case, could evolve after capture under repeated Lorentz force, which uses no propellant. However, tether performance depends on ambient conditions $\left(\bar{E}_{m}, \bar{B}\right.$, and electron plasma density $N_{e}$ ) as well as on the orbit geometry assumed equatorial.

The Jovian region of interest in this paper lies in the so-called inner magnetosphere, where the field $B$ is basically produced by currents inside Jupiter, limited measurements determining dominant terms in the multipole expansion. A simple no-tilt nooffset dipole model will be adopted in our analysis. We shall use the Divine-Garrett model of the thermal Jovian plasma, basically constructed from Pioneer 10 and 11 and Voyager 1 and 2 in situ data, supplemented by Earth-based observations of synchrotron emission [29]. Plasmasphere, which is independent of longitude in this model, and Io torus are modeled separately; the torus model will only be required for the mission study of [28], where radiation doses are also determined. Only the plasma density profile, which has a simple analytical representation, is actually involved in the present calculations. Updates on the D/G model have been limited to the $I o$ torus or just to the radiation population. 
SC capture requires the Lorentz drag to make a minimum work $\left|W_{C}\right|$ to take the orbital energy from a positive value $M_{\mathrm{SC}} v_{\infty}^{2} / 2$ in the incoming hyperbolic orbit to some negative value; the mass of the tether $m_{t}$ will be considered included in the SC mass $M_{\mathrm{SC}}$. For the given incoming perijove radius $r_{p}$, the greater is the work $\left|W_{C}\right|$, the smaller are apojove radius and eccentricity $e_{1}$ in the first orbit following capture. A calculation of $\left|W_{C}\right|$ must take into account that the SC will follow an orbit far from circular during capture. This involves considering ambient conditions that vary along the orbit, a motional field that has a complex behavior (the condition $r<a_{s}$ for Lorentz drag not strictly applying for elliptical or inclined orbits), and a tether oriented neither normal to the trajectory nor along the local vertical. The weak gravity gradient in Jupiter will require setting the tethered system to spin in the orbital plane, as later discussed (see Section V).

Fortunately, the incoming orbit starts barely hyperbolic and, for realistic estimates of $\left|W_{C}\right|$ as found here, ends barely elliptic. With the Lorentz force only acting around perijove, the energy per unit mass in the incoming hyperbolic orbit and the successive elliptical orbits only depends on eccentricity

$$
\varepsilon=\frac{-\mu_{J}}{2 r_{p}}(1-e)
$$

For $\varepsilon_{h}=1 / 2 v_{\infty}^{2}$, the hyperbolic eccentricity will be just above unity

$$
e_{h}-1=\frac{v_{\infty}^{2} r_{p}}{\mu_{J}} \equiv \widetilde{v}_{\infty}^{2} \frac{r_{p}}{R_{J}}
$$

with $\widetilde{v}_{\infty}^{2} \approx 0.018$ for $v_{\infty} \approx 5.64 \mathrm{~km} / \mathrm{s}$ (case of Hohmann transfer from Earth). We make the ansatz that the SC is barely captured, with $e_{1}$ after capture just below unity

$$
\frac{e_{h}-e_{1}}{e_{h}-1}=\frac{-\Delta \varepsilon}{v_{\infty}^{2} / 2}=\frac{-W_{c}}{M_{\mathrm{SC}} v_{\infty}^{2} / 2}=O(1)
$$

This means that the orbit is hardly affected locally and that we can ignore all changes except the dramatic faraway effect of having it changed from open to closed. We will consider throughout capture a parabolic $(e=1)$ orbit, which is entirely determined by its perijove radius $r_{p}$, assuming no longitudinal dependence on ambient conditions.

With the Jovian no-tilt magnetic field pointing south at the equator $(-B \bar{k}, B>0)$ and taking a unit vector $\bar{u}$ along the tether, from the cathodic to the anodic end (the direction of conventional current), Lorentz force and corresponding mechanical power read [Fig. 2(a)]

$$
\begin{aligned}
\bar{F}_{L} & =L \bar{I}_{\mathrm{av}} \times \bar{B}=L B I_{\mathrm{av}}(\bar{k} \times \bar{u}) \\
\dot{W} & =\bar{F}_{L} \cdot \bar{u}_{t} v=v L B I_{\mathrm{av}} \bar{k} \cdot\left(\bar{u} \times \bar{u}_{t}\right)=-v L B I_{\mathrm{av}} \sin \left(\alpha_{E}+\varphi\right)
\end{aligned}
$$

with $\alpha_{E}$ and $\varphi$ as shown in the figure.

For a bare tether, the Lorentz force involves the current averaged over the tether length $I_{\mathrm{av}}$, which will depend on all impedances in the tether circuit. The hollow cathode contact impedance and the radiation impedance for current closure in

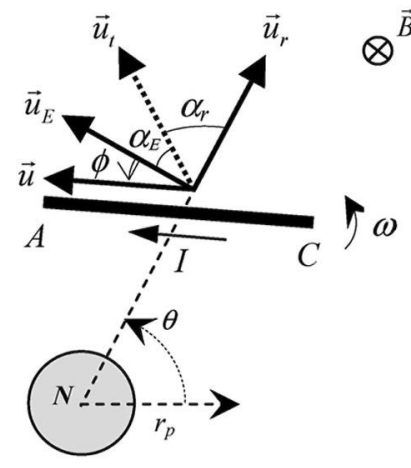

(a)

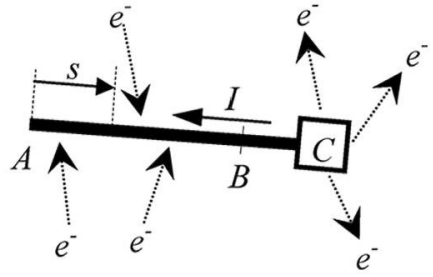

(b)
Fig. 2. (a) Relative positions of unit vectors for spinning tether $\bar{u}$, motional electric field $\bar{u}_{E}$, orbit tangent $\bar{u}_{t}$, and radius vector $\bar{u}_{r}$, at true anomaly $\theta$ in a parabolic equatorial orbit. $\mathrm{A}$ and $\mathrm{C}$ are anodic and cathodic ends, respectively. Ends exchange roles every half tether turn. (b) Sketch of bare-tether operation. Electrons are collected over an anodic segment from end A to some point B. Voltage bias is negative to the right of $\mathrm{B}$; ion collection over the cathodic segment $\mathrm{BC}$ comes out to be negligible. Electrons are ejected at the hollow cathode at $\mathrm{C}$. The hollow cathode at end $\mathrm{A}$ is off.

the Jovian plasma are negligible; also, we assume that any power-load output impedance is low during capture. With $\bar{u}_{E}$, the unit vector along the motional electric field, and writing $\bar{v}^{\prime} \equiv \bar{v}-\bar{v}_{\mathrm{pl}}$, we have

$$
E_{m} \equiv \bar{E}_{m} \cdot \bar{u}=v^{\prime} B \cos \varphi>0 .
$$

We then introduce the current $i_{\mathrm{av}}$ normalized with the shortcircuit value

$$
I_{\mathrm{av}} \equiv i_{\mathrm{av}} \sigma_{c} E_{m} w h=i_{\mathrm{av}} \sigma_{c} w h v^{\prime} B \cos \varphi
$$

where $\sigma_{c}$ is tether conductivity and $i_{\mathrm{av}}$ will itself be a function of $\cos \varphi$, as shown in the next section. Assuming here a fast enough tether spin $\omega$, we can average (7) over the angle $\varphi$ at fixed $r$, using $\bar{v}^{\prime} \perp \bar{u}_{E}$ and Fig. 2(a) to write the relative velocity along the orbit as $v_{t}^{\prime} \equiv \bar{v}^{\prime} \cdot \bar{u}_{t}=v^{\prime} \sin \alpha_{E}$, and find

$$
\langle\dot{W}\rangle=-\sigma_{c} L w h B^{2} v v_{t}^{\prime}\left\langle i_{\mathrm{av}} \cos ^{2} \varphi\right\rangle
$$

where the bracket stands for $\varphi$ average; note that it suffices considering the range $\cos \varphi>0$.

From Fig. 2(a), we can write

$$
\bar{v}^{\prime}=\left(v-\Omega_{J} r \sin \alpha_{r}\right) \bar{u}_{t}+\Omega_{J} r \cos \alpha_{r} \bar{u}_{n}
$$

with $\bar{u}_{n}$ along the normal to the trajectory. The energy and conic equations for a parabolic orbit read

$$
v^{2}=2 \mu_{J} / r \quad 1+\cos \theta=2 r_{p} / r .
$$

Setting $v r \sin \alpha_{r}=v_{p} r_{p}$ from the conservation of angular momentum and using (11) and (12a) and the relation $\Omega_{J}^{2} a_{s}^{3}=\mu_{J}$, we find

$$
v v_{t}^{\prime}=v^{2}\left(1-r / r_{M}\right)
$$


with the radius $r_{M}$, where relative tangential velocity $v_{t}^{\prime}$ and drag vanish, reading

$$
r_{M}\left(r_{p}\right) \equiv a_{s} \sqrt{2 a_{s} / r_{p}}
$$

Note that there exists a drag arc, $r_{M}>r_{p}$, for $r_{p}<2^{1 / 3} a_{s} \approx$ $2.82 R_{J}$. The drag arc reaches beyond $a_{s}$ whenever it exists. This is due to both parabolic velocities being $\sqrt{ } 2$ times greater than corresponding circular velocities and corotation being at an angle to the $\mathrm{SC}$ velocity.

Next, using (12b) and Barker's equation giving time $t$ from perijove pass versus true anomaly $\theta$ for a parabolic orbit, we find

$$
\frac{3}{2} \frac{v_{p}}{r_{p}} t=\left(2+\frac{r}{r_{p}}\right) \sqrt{\frac{r-r_{p}}{r_{p}}} \Rightarrow d t=\frac{r d r}{v_{p} \sqrt{r_{p}\left(r-r_{p}\right)}} .
$$

It follows that the time the $\mathrm{SC}$ takes in describing the drag arc is

$$
\begin{aligned}
\Delta t & =2 \frac{2 r_{p}}{3 v_{p}}\left(2+\frac{r_{M}}{r_{p}}\right) \sqrt{\frac{r_{M}-r_{p}}{r_{p}}} \\
& \approx \frac{r_{M}+2 r_{p}}{r_{p}} \sqrt{\frac{r_{M}-r_{p}}{r_{p}}} \times 2.11 \mathrm{~h}
\end{aligned}
$$

where we used $\left(r_{p} / a_{s}\right)^{1 / 2} v_{p}=v_{s} \approx 39.8 \mathrm{~km} / \mathrm{s}$. The $\varphi$ average in (10) will thus require that the tether spin period be at most a fraction of an hour.

Then, integrating over the time on the full drag arc, we obtain

$$
\begin{aligned}
W_{C}=\int\langle\dot{W}\rangle d t=-2 \sigma_{c} L w h \int_{r_{p}}^{r_{M}} & \frac{B^{2} v^{2} r d r}{v_{s} \sqrt{a_{s}\left(r-r_{p}\right)}} \\
& \times \frac{r_{M}-r}{r_{M}}\left\langle i_{\mathrm{av}} \cos ^{2} \varphi\right\rangle .
\end{aligned}
$$

Using

$$
B=B_{s} a_{s}^{3} / r^{3} \quad\left(B_{s} \approx 0.38 \mathrm{G}\right)
$$

and (5), we finally find

$$
\frac{-2 W_{C}}{m_{t} v_{\infty}^{2}}=\frac{e_{h}-e_{1}}{e_{h}-1} \frac{M_{\mathrm{SC}}}{m_{t}}=\widetilde{B}_{s}^{2} S
$$

with

$$
\begin{aligned}
\widetilde{B}_{s}^{2} & \equiv \frac{\sigma_{c} B_{s}^{2} a_{s} v_{s}}{2^{5 / 6} \rho_{t} v_{\infty}^{2}} \\
S & \equiv \widetilde{r}_{M}^{8 / 3} \int_{1}^{\widetilde{r}_{M}} \frac{\left(\widetilde{r}_{M}-\widetilde{r}\right) d \widetilde{r}}{\widetilde{r}^{6} \sqrt{\widetilde{r}-1}}\left\langle 2 i_{\mathrm{av}} \cos ^{2} \varphi\right\rangle \\
\widetilde{r} & =r / r_{p}, \quad \widetilde{r}_{M} \approx 4.74\left(R_{J} / r_{p}\right)^{3 / 2} .
\end{aligned}
$$

Note that efficient capture, i.e., high dimensionless parameter $\widetilde{B}_{s}$, is favored by a strong planetary magnetic field and a high tether conductivity/density $\left(\sigma_{c} / \rho_{t}\right)$ ratio.

\section{BARE-TETher CAPTURE}

We now compute the average current to the bare tape using the OML current law and the Divine-Garrett model for the thermal plasma in the equatorial plasmasphere. The electron current $I(s)$ in a bare tether starts from zero at the anodic end A and increases with distance $s$ from A [Fig. 2(b)], as electrons collected under the OML law

$$
\frac{d I}{d s}=\frac{2 w}{\pi} e N_{e} \sqrt{\frac{2 e \Delta V}{m_{e}}} \quad\left(0<s<s_{B}\right)
$$

pile up over the segment at bias $\Delta V$ positively with respect to the local plasma [26]. The difference between ohmic and induced-voltage drops makes tether bias decrease at a rate

$$
\frac{d \Delta V}{d s}=\frac{I}{\sigma_{c} h w}-E_{m}
$$

with the rate itself decreasing as current increases and vanishing where, and if, the current reaches the short-circuit value $\sigma_{c} E_{m} w h$.

There exists a point $\mathrm{B}$ where $\Delta V$ vanishes because bias must be negative at end $\mathrm{C}$ to allow a hollow cathode to eject electrons. Equations (20) and (21) yield a first integral for the $\mathrm{AB}$ segment

$$
\psi^{3 / 2}+2 i-i^{2}=\psi_{A}^{3 / 2}=2 i_{B}-i_{B}^{2}
$$

where we used boundary conditions $i_{A}=\psi_{B}=0$ and defined $\psi \equiv \Delta V / E_{m} L^{*}, i \equiv I / \sigma_{c} E_{m} w h$, and where $L^{*}$ is a characteristic length gauging the bare-tether collection impedance against the tether ohmic resistance [26]

$$
\begin{aligned}
\frac{4}{3} e N_{e} \frac{2 L^{*}}{\pi} \sqrt{\frac{2 e E_{m} L^{*}}{m_{e}}} & \equiv \sigma_{c} E_{m} h \\
& \Rightarrow L^{*} \propto E_{m}^{1 / 3}\left(\frac{\sigma_{c} h}{N_{e}}\right)^{2 / 3} .
\end{aligned}
$$

Using (22) in (21), one finds

$$
\begin{aligned}
\frac{s}{L^{*}} & =\int_{\psi}^{\psi_{A}} \frac{d \psi^{\prime}}{\sqrt{1-\psi_{A}^{3 / 2}+\psi^{\prime 3 / 2}}} \\
& \leq \frac{s_{B}}{L^{*}} \\
& =\int_{0}^{\psi_{A}} \frac{d \psi^{\prime}}{\sqrt{1-\psi_{A}^{3 / 2}+\psi^{\prime 3 / 2}}} .
\end{aligned}
$$

For just drag or thrust, with no power load at $\mathrm{C}$, its negative bias is the hollow-cathode bias, which is comparatively very small. Setting $\left|\psi_{C}\right| \approx 0$, one readily finds the average current by integrating (21) over the entire tether length

$$
1-i_{\mathrm{av}}=\psi_{A} / \hat{L}, \quad\left(\hat{L} \equiv L / L^{*}\right) .
$$

We now obtain a law $i_{\text {av }}(\hat{L})$ by determining $\psi_{A}(\hat{L})$.

Along the negatively biased segment $\mathrm{BC}$, electrons leak out at the OML rate of impact of ions, which leave as neutrals [Fig. 2(b)]

$$
d I / d s=-(2 w / \pi) e N_{e} \sqrt{2 e|\Delta V| / m_{i}}, \quad\left(s_{B}<s<L\right) .
$$




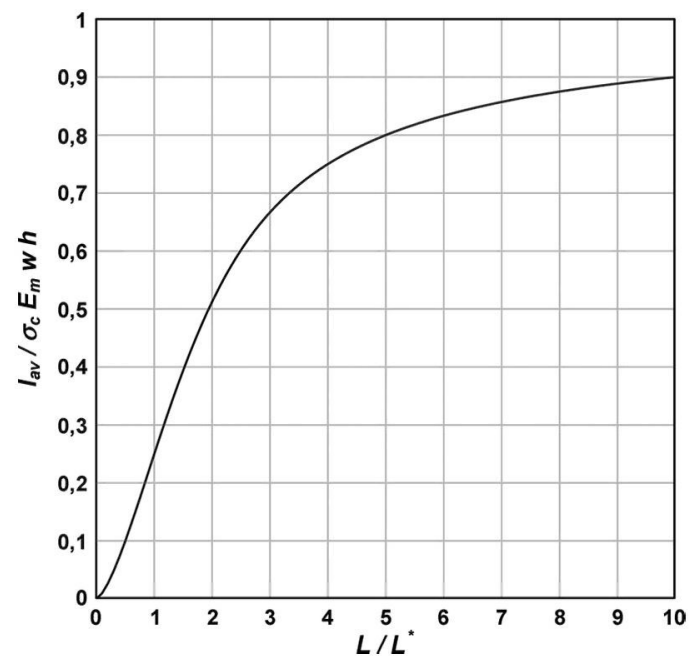

Fig. 3. Normalized average tether current $i_{\text {av }}$ versus normalized tether length $\hat{L}$, given in (28).

Current collection over the segment $\mathrm{BC}$ is then doubly small because the mass ratio $m_{e} / m_{i}$ is small and because we have $|\Delta V|<\left|\Delta V_{C}\right|$ throughout, allowing one to write

$$
I \approx I_{B} \approx I_{C}, \quad\left(s_{B}<s<L\right) .
$$

Consider now a current $i_{B}=1$ giving values $\psi_{A}=1$ and $s_{B}=4 L^{*}$ in (22) and (24). We now have both $\psi$ and $d \psi / d s$ vanishing at $B$, with (26) and (21) yielding $\psi=$ const $=0$ and $i=$ const $=1$ throughout the segment $\mathrm{BC}$ whatever its length. We thus have $\psi_{A}=1$ for any value $L>4 L^{*}$.

Next, consider values $i_{B}<1\left(\psi_{A}<1, s_{B}<4 L^{*}\right)$. Integrating (21) from $\mathrm{B}$ to $\mathrm{C}$ would give $0 \approx\left(i_{B}-1\right) \times\left(L-s_{B}\right)$, requiring a negligibly short cathodic segment $\mathrm{BC}, L-s_{B} \approx 0$ in Fig. 2(b). For any length, $L<4 L^{*}$ we find $s_{B} \approx L$, the ratio $L / L^{*}$ then given in terms of the bias at A by (24). Thus, we finally find $\psi_{A}(\hat{L})$

$$
\int_{0}^{\psi_{A}} \frac{d \psi}{\sqrt{1-\psi_{A}^{3 / 2}+\psi^{3 / 2}}}=\hat{L} \leq 4, \quad \psi_{A}=1 ; \quad \hat{L} \geq 4 .
$$

The use of (25) then yields $i_{\text {av }}(\hat{L})$, which is shown in Fig. 3 [30].

Finally, from (23), we have

$$
\begin{aligned}
\frac{L}{L^{*}} & \equiv \hat{L}\left(\cos \varphi, \widetilde{r}, \Lambda, \widetilde{r}_{M}\right) \\
& \equiv\left(\frac{2^{7 / 2} N_{e}}{3 \pi \sigma_{c} h}\right)^{2 / 3} \frac{e L}{\left(m_{e} v^{\prime} B|\cos \varphi|\right)^{1 / 3}} \\
& \approx \frac{\Lambda}{|\cos \varphi|^{1 / 3}} \frac{\widetilde{r}^{7 / 6}\left(N_{e} / N_{s}\right)^{2 / 3}}{\widetilde{r}_{M}^{4 / 9}\left(\tilde{r}_{M}^{2}+\widetilde{r}^{3}-2 \widetilde{r}_{M} \tilde{r}\right)^{1 / 6}}
\end{aligned}
$$

where

$$
\Lambda \equiv \frac{2^{49 / 18}}{(3 \pi)^{2 / 3}}\left(\frac{N_{s}}{\sigma_{c} h}\right)^{2 / 3} \frac{e L}{\left(m_{e} v_{s} B_{s}\right)^{1 / 3}}
$$

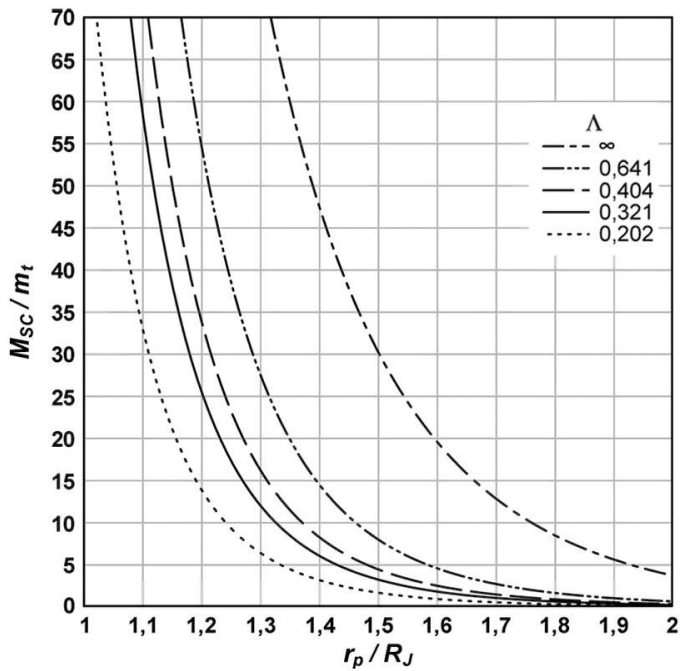

Fig. 4. Full mass of SC in Hohmann transfer from Earth that can be captured into a barely elliptical Jovian orbit $\left[-\Delta e=e_{h}-1\right.$ in (32)] by an aluminum foil of mass $m_{t}$ versus perijove of capture. The parameter $\Lambda$ depends on foil length and thickness as given in (33b). Half that mass could be captured into an elliptical orbit of eccentricity of about 0.98 .

and $N_{e} / N_{s}$ is given in terms of $\widetilde{r}, \widetilde{r}_{M}$ by the thermal plasma density law in the Divine-Garrett model of the plasmasphere at zero latitude [29]

$$
\frac{N_{e}}{N_{s}}\left(\widetilde{r}, \widetilde{r}_{M}\right)=\exp \left(\frac{r_{0}}{r}-\frac{r_{0}}{a_{s}}\right) \equiv \exp \left(\frac{2.72 \widetilde{r}_{M}^{2 / 3}}{\widetilde{r}}-3.43\right)
$$

with $r_{0}=7.68 R_{J}$ and $N_{s}=1.44 \times 10^{2} \mathrm{~cm}^{-3}$. Also, from (8) and (11), we wrote

$$
E_{m}=\sqrt{v^{2}+\Omega_{J}^{2} r^{2}-2 \Omega_{J} r_{p} v_{p}} B \cos \varphi
$$

which was used in (28).

With (25), (27a, b), and (28) giving $i_{\mathrm{av}}\left[\hat{L}\left(\cos \varphi, \Lambda, \widetilde{r}, \widetilde{r}_{M}\right)\right]$ and (19b) for $\widetilde{r}_{M}$, we have $S \equiv S\left(\Lambda, r_{p} / R_{J}\right)$ after $\varphi$-averaging and $\tilde{r}$ integration in (18) for use in (16)

$$
\frac{M_{\mathrm{SC}}}{m_{t}}=\frac{e_{h}-1}{-\Delta e} \widetilde{B}_{s}^{2} S\left(\Lambda, \frac{r_{p}}{R_{J}}\right) .
$$

In case of an aluminum foil and Hohmann transfer, we find

$$
\widetilde{B}_{s}^{2} \approx 2.11 \quad \Lambda \approx 0.200 \frac{L}{50 \mathrm{~km}}\left(\frac{0.05 \mathrm{~mm}}{h}\right)^{2 / 3} .
$$

Fig. 4 uses the values in (33a) to represent the SC-to-tether mass ratio in (16) in case the first orbit after capture is barely elliptic, $|\Delta e|=e_{h}-1$, i.e., $1-e_{1}=0^{+}$.

The limit $\Lambda \rightarrow \infty$ corresponds to dominant ohmic effects, leading to $i_{\mathrm{av}} \rightarrow 1$. We find a numerical value $\max S=$ $S(\infty, 1) \approx 178$, which is independent of the ambient plasma density law. Because the surface magnetic field is about 20 times smaller for Saturn than for Jupiter and all parameters other than $B_{s}$ entering in (17) are comparable for the two planets, the factor $\widetilde{B}_{s}^{2}$ in (16) will be smaller for Saturn by two 


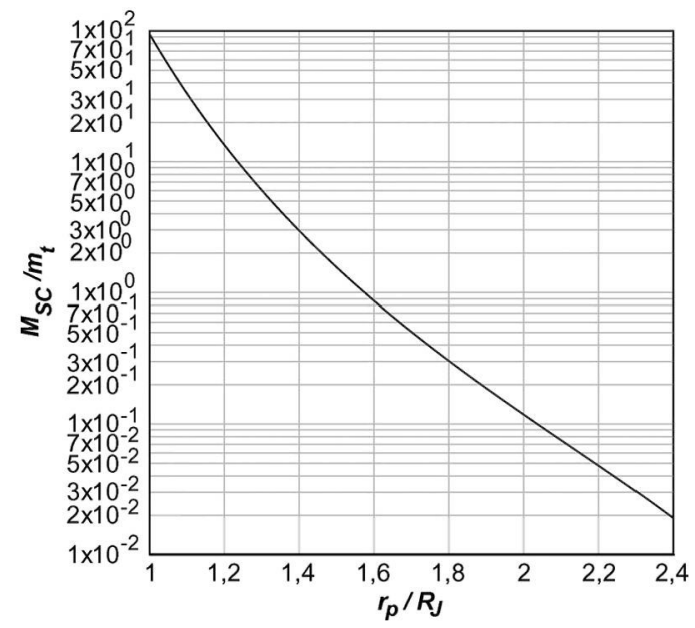

Fig. 5. Full mass of SC in Hohmann transfer from Earth that can be captured into a barely elliptical Jovian orbit [with eccentricity $e_{1} \approx 1^{-}$in (34a)] under conditions of negligible ohmic effects, for $L=50 \mathrm{~km}$ and $h=0.05 \mathrm{~mm}$. The mass capture ratio scales as $L^{3 / 2} / h$.

and a half orders of magnitude. This will make tether capture in Saturn very difficult.

In the opposite limit of small $\Lambda$ and, thus, small $\hat{L}$, we have $i_{\mathrm{av}} \approx 0.3 \hat{L}^{3 / 2}$ [30]. As shown in Fig. 3 , this $i_{\mathrm{av}}$ behavior is reasonably accurate up to $\hat{L} \approx 1$. Because $\hat{L}^{3 / 2} \propto \Lambda^{3 / 2}$ varies as $1 / \sigma_{c} h$, the current $I_{\mathrm{av}}=i_{\mathrm{av}} \sigma_{c} h w E_{m}$ is then independent of conductivity (and foil thickness). Equation (33b) then shows that tether foils with reasonable values of length and thickness will be free of ohmic effects. Again, with $S \sim \Lambda^{3 / 2}$ in (18) and $\widetilde{B}_{s}^{2} \propto \sigma_{c}$, the mass ratio in (32) takes a simple form, similarly independent of conductivity and convenient for design considerations. Using (28) and (29) and the small- $\Lambda i_{\mathrm{av}}$ law in (18), we find

$$
\begin{aligned}
\frac{-\Delta e}{e_{h}-1} \frac{M_{\mathrm{SC}}}{m_{t}} & =0.80 \frac{m_{e} N_{s} a_{s} \sqrt{v_{s}}\left(L e B_{s} / m_{e}\right)^{3 / 2}}{h \rho_{t} v_{\infty}^{2}} \Sigma\left(\frac{r_{p}}{R_{J}}\right) \\
& \approx 0.15\left(\frac{L}{50 \mathrm{~km}}\right)^{3 / 2} \frac{0.05 \mathrm{~mm}}{h} \Sigma\left(\frac{r_{p}}{R_{J}}\right) \\
\Sigma & \equiv \int_{1}^{\widetilde{r}_{M}} \frac{\widetilde{r}_{M}^{2} d \widetilde{r} / 2^{5 / 4} \widetilde{r}^{17 / 4}}{\left(\widetilde{r}_{M}^{2}+\widetilde{r}^{3}-2 \widetilde{r}_{M} \widetilde{r}\right)^{1 / 4}} \frac{\widetilde{r}_{M}-\widetilde{r}}{\sqrt{\widetilde{r}-1}} \frac{N_{e}}{N_{s}}\left(\widetilde{r}, \widetilde{r}_{M}\right)
\end{aligned}
$$

as against (32); in (34a), we used an aluminum foil and Homann transfer. Fig. 5 shows the SC-to-tether mass ratio from (34) in case the first orbit after capture is barely elliptic for an aluminum foil and Hohmann transfer.

The use of (33b) in Fig. 4 shows that an aluminum foil of thickness $0.05 \mathrm{~mm}$ and length of about $80 \mathrm{~km}$ can capture an $\mathrm{SC}$ over six times its mass from a Hohmann orbit with perijove at $1.4 R_{J}$ into an orbit that is barely elliptical, $e_{1}=1-0^{+}$. For perijove at $1.3 R_{J}, L$ could be as short as $50 \mathrm{~km}$; an aluminum foil with dimensions $L=50 \mathrm{~km}, h=0.05 \mathrm{~mm}$, and $w=3 \mathrm{~cm}$, its mass $m_{t}$ being about $200 \mathrm{~kg}$, could capture a full SC mass of $1300 \mathrm{~kg}$. With $e_{h} \approx 1.023$ in (4), cutting $M_{\mathrm{SC}} / m_{t}$ by half would yield $e_{1} \approx 0.977$. Here, ohmic effects are weak, with the use of Fig. 5 yielding similar results.

\section{Local Quasi-STEAdy Thermal Balance}

We now consider whether efficient SC capture by thin long tapes at low perijove might result in fast intense heating. The energy equation determining the temperature $T(s)$ of an element of tether length $\delta s$ is [Fig. 2(b)]

$$
w h \delta s \rho_{t} c_{t} \frac{\partial T}{\partial t}=\dot{w}_{\mathrm{th}} \delta s-2 w \delta s \varepsilon_{t} \sigma_{B} T^{4}+w h \delta s K_{t} \frac{\partial^{2} T}{\partial s^{2}}
$$

where $c_{t}, \varepsilon_{t}$, and $K_{t}$ are the tether specific heat, emissivity, and thermal conductivity, respectively, and $\sigma_{B}$ is the Stefan-Boltzmann constant. We are ignoring any small heating from the natural Jovian environment. Also, the last term in (35) can be readily ignored when compared with the first term, the thermal diffusivity for aluminum $K_{t} / \rho_{t} c_{t} \approx 8.7 \times 10^{-5} \mathrm{~m}^{2} / \mathrm{s}$, being many orders of magnitude smaller than any reasonable value for $L^{2} \omega$.

We now calculate heating at the perijove during capture, which will make for the greatest heating rate. We will take into account the rotation of the tether as it heats or cools but assume its position fixed at the perijove as in the dynamical analyses of Sections II and III, making for a slightly conservative estimate. The heating power

$$
\dot{w}_{\mathrm{th}} \delta s=\frac{I^{2} \delta s}{\sigma_{c} w h}+\Delta V \frac{d I}{d s} \delta s
$$

is made of two contributions, one due to ohmic dissipation from the current $I(s)$ flowing through the element $\delta s$ and a second contribution due to the energy brought up to the element by the impact of collected electrons. Integrating (36) over the entire length of the tether, using (21) and the values $I_{A}=0$ and $\Delta V_{C} \approx 0$, yields

$$
\int_{0}^{L} \dot{w}_{\mathrm{th}} d s=I_{\mathrm{av}} E_{m} L
$$

with the two terms in (36) thus adding up to the entire power from the Lorentz force.

In the realistic weak ohmic-effect limit, we ignore the first terms in the right-hand side of both (21) and (36). Equations (21) and (20) then yield

$$
\Delta V(s)=E_{m}(L-s) \quad \delta I(s)=\frac{2 w}{\pi} e N_{e} \sqrt{\frac{2 e}{m_{e}} E_{m}(L-s)} \delta s .
$$

Equation (36), in turn, reads

$$
\dot{w}_{\mathrm{th}}(s) \delta s=\Delta V \delta I=e N_{e} \frac{2 w}{\pi} \sqrt{\frac{2 e E_{m} L}{m_{e}}} E_{m} L\left(1-\frac{s}{L}\right)^{3 / 2} \delta s .
$$

Using (30) and (31) at perijove to evaluate the heating power in (38), the energy equation [(35)] reads

$$
\begin{array}{rl}
\frac{\partial T}{\partial t}=\frac{m_{e} N_{s}}{\pi c_{t} \rho_{t} h}\left(\frac{2 e v_{s} B_{s} L}{m_{e}}\right)^{3 / 2} & G\left(\tilde{r}_{M}\right)\left(1-\frac{s}{L}\right)^{3 / 2} \\
& \times \cos ^{3 / 2} \varphi-\frac{2 \varepsilon_{t} \sigma_{B}}{h c_{t} \rho_{t}} T^{4}
\end{array}
$$


where

$$
G \equiv \frac{\widetilde{r}_{M}^{2}\left(\widetilde{r}_{M}-1\right)^{3 / 2}}{2^{7 / 4}} \times \frac{N_{e}}{N_{s}}\left(\widetilde{r}=1, \widetilde{r}_{M}\right)
$$

Using $d \varphi=\omega d t$ and defining

$$
\begin{aligned}
\widetilde{T} & \equiv \frac{T}{T_{0}} \quad T_{0}^{4} \equiv \frac{m_{e} N_{s}}{2 \pi \sigma_{B} \varepsilon_{t}}\left(\frac{e v_{s} B_{s} L}{m_{e}}\right)^{3 / 2} G\left(\widetilde{r}_{M}\right) \\
\delta & =\frac{\pi^{3 / 4} c_{t} \rho_{t} h \omega}{\left(2 \sigma_{B} \varepsilon_{t}\right)^{1 / 4}\left[m_{e} N_{s} G\left(\widetilde{r}_{M}\right)\right]^{3 / 4}}\left(\frac{m_{e}}{e v_{s} B_{s} L}\right)^{9 / 8}
\end{aligned}
$$

(39) becomes

$$
\frac{\partial \widetilde{T}}{\partial \varphi} \delta=\left(2 \frac{L-s}{L}\right)^{3 / 2}|\cos \varphi|^{3 / 2}-\widetilde{T}^{4} .
$$

Note that the energy equation [(43)] is dependent on the tether point considered. There are two simple limiting cases corresponding to values $s=L / 2$ and $s=0(L)$. In this second case, a value $s=0$ for $-\pi / 2<\varphi<\pi / 2$ corresponds to a value $s=L$ for $\pi / 2<\varphi<3 \pi / 2$, the energy equation then reading

$$
\begin{aligned}
& \frac{\partial \widetilde{T}}{\partial \varphi} \delta=2^{3 / 2} \cos \varphi^{3 / 2}-\widetilde{T}^{4}, \quad-\pi / 2<\varphi<\pi / 2 \\
& \frac{\partial \widetilde{T}}{\partial \varphi} \delta=-\widetilde{T}^{4}, \quad \pi / 2<\varphi<3 \pi / 2
\end{aligned}
$$

whereas, in the first case, a single equation is valid throughout

$$
\frac{\partial \widetilde{T}}{\partial \varphi} \delta=|\cos \varphi|^{3 / 2}-\widetilde{T}^{4}, \quad-\pi / 2<\varphi<3 \pi / 2 .
$$

For a small rise time $\delta$, the temperature would follow quasisteadily the changing angle in $(44 a, b)$ and (45). Its absolute maximum would then occur at the tether anodic end in (44a), yielding $\widetilde{T}_{\max }=2^{3 / 8}$. Consider values $L=80 \mathrm{~km}$ and $r_{p}=$ $1.4 R_{J}$; an emissivity $\varepsilon_{t}=0.8$ would now yield $T_{0}=510 \mathrm{~K}$. Maximum temperature would thus be

$$
T_{\max }=2^{3 / 8} T_{0}=661 \mathrm{~K} .
$$

With the aforementioned parameter values, a $30-\mathrm{min}$ spin, and $h=0.05 \mathrm{~mm}$, the rise time would indeed be small, $\delta \approx 0.035$.

The absolute maximum at the anodic end might be reduced by reducing the temperature $T_{0}$ or increasing (substantially) the rise time. From the scaling laws

$$
\begin{aligned}
\delta & \propto \frac{\rho_{t} c_{t} h \omega}{\varepsilon_{t}^{1 / 4} L^{9 / 8}\left[G\left(\widetilde{r}_{M}\right)\right]^{3 / 4}} \\
T_{0} & \propto \frac{L^{3 / 8}}{\varepsilon_{t}^{1 / 4}} G\left(\widetilde{r}_{M}\right)^{1 / 4}
\end{aligned}
$$

both effects can be attained by reducing the tether length. Notice, however, that reducing the tether length (thus reducing the parameter $\Lambda$ ), makes SC capture harder. This may be compensated by placing the perijove closer to Jupiter. We find little net effect in changing to values $L=50 \mathrm{~km}$ and $r_{p}=1.3 R_{J}$

$$
\delta \approx 0.030 \quad T_{0}=536 \mathrm{~K} \Rightarrow T_{\max }=695 \mathrm{~K} . \quad(49 \mathrm{a}, \mathrm{b})
$$

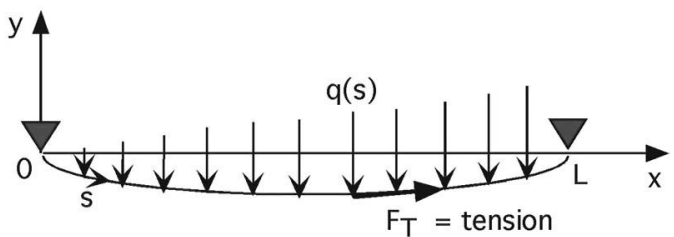

Fig. 6. Schematics of tether under lateral load and tension $F_{T}$.

Independently, just the rise time could be increased by using a faster spin; as shown in the next section, however, too high a spin rises new issues. Moving from a 30 - to 12 -min spin period, for example, with $L=50 \mathrm{~km}$ and $r_{p}=1.3 R_{J}$, still yields a low $\delta(\approx 0.076)$.

\section{Bowing And Tensile STRESS Constraints}

Avoiding too large tether bowing due to the strong lateral Lorentz force at capture may require a corresponding strong tension, which might be attained by tether spinning. For a simple conservative estimate of bowing, consider the classical equation of statics for a rope under lateral load per unit length $q$ and supported at the two ends

$$
\frac{d^{2} y}{d s^{2}}=\frac{q}{F_{T}}
$$

where $y$ is the lateral deflection of the rope and $F_{T}$ is the tensile force (Fig. 6). Here, the distributed lateral load $q(s)$ is the Lorentz force per unit tether length

$$
q(s) \equiv \delta F_{L} / \delta s=B I(s)
$$

and $s$ is measured from (for example) the anodic end as in Fig. 2(b). We are assuming that the tension is large enough to keep deflections small.

In the ohmic-dominated limit, we would have $I=$ const $=$ $\sigma_{c} E_{m} w h$ over near the entire tether length. Integrating (50) twice with boundary conditions $y(0)=y(L)=0$, yields the classical result for uniform load distribution, with maximum deflection $|y|_{\max } / L=F_{L} / 8 F_{T}$ (at midpoint, $s=L / 2$ ). The deflection in the opposite limit, more appropriate to the Jovian case as noticed in Section III, is similar. We use (37b) to find the current distribution along the tether length

$$
\frac{I(s)}{I_{\max }}=1-\left(1-\frac{s}{L}\right)^{3 / 2} \quad I_{\mathrm{av}}=\frac{3}{5} I_{\max } .
$$

Using $F_{L}=I_{\mathrm{av}} B L$ from (6), for $q(s)$ in (50), we have

$$
q(s)=\frac{5}{3} \frac{F_{L}}{L}\left[1-\left(1-\frac{s}{L}\right)^{3 / 2}\right] .
$$

A straightforward integration yields a maximum deflection (at $s \approx 0.564 L$ )

$$
\frac{|y|_{\max }}{L}=\frac{F_{L}}{7.6 F_{T}} .
$$


Requiring the aforementioned percent deflection not to exceed some small value, for example, 0.1 , sets a condition for minimum tension

$$
F_{T}>1.31 F_{L} .
$$

Again, using (37b) to determine $I_{\mathrm{av}}$ for the no-ohmic effect limit, we find

$$
F_{L}=B L \frac{4}{5} \frac{w L}{\pi} e N_{e} \sqrt{\frac{2 e L}{m_{e}} v^{\prime} B|\cos \varphi|}
$$

which is maximum at perijove with $|\cos \varphi|=1$

$$
\begin{aligned}
\max F_{L}=\frac{4}{5 \pi} w L^{2} e & N_{s} B_{s} \sqrt{\frac{2 e v_{s} B_{s} L}{m_{e}}} \\
& \times \frac{\widetilde{r}_{M}^{8 / 3} \sqrt{\widetilde{r}_{M}-1}}{2^{19 / 12}} \times \frac{N_{e}}{N_{s}}\left(\widetilde{r}=1, \widetilde{r}_{M}\right) .
\end{aligned}
$$

For $L=50 \mathrm{~km}, r_{p}=1.3 R_{J}$, for example, we find

$$
\max F_{L} \approx 312 \mathrm{~N} \times w / 3 \mathrm{~cm} .
$$

The gravity-gradient force provides the tension required to keep a tether taut in circular orbit with angular rate $\Omega$ around a planet in situations with small lateral loads. The standard formula for the gravity-gradient tensile force on a tether kept straight along the local vertical while orbiting under negligible lateral force is

$$
F_{T}=\frac{3 \Omega^{2} L M_{\mathrm{SC}}}{4}
$$

where a massless tether and equal masses $M_{\mathrm{SC}} / 2$ at either end were considered. Two thirds of the aforementioned tension arise from the fact that gravitational forces decrease as the inverse square of distance from the planet center. Taking into account tether mass, with equal end masses $\left(M_{\mathrm{SC}}-m_{t}\right) / 2$, yields a small correction that makes the tension vary with distance $s^{\prime} \equiv$ $(L / 2)-s$ from midtether in Fig. 2(b)

$$
\begin{aligned}
F_{T} & =\frac{3 \Omega^{2} L M_{\mathrm{SC}}}{4}\left[1-\frac{m_{t}}{2 M_{\mathrm{SC}}}\left\{1+\left(\frac{s^{\prime}}{L / 2}\right)^{2}\right\}\right] \\
& \approx \frac{3 \Omega^{2} L M_{\mathrm{SC}}}{4}\left[1-\frac{2 m_{t}}{3 M_{\mathrm{SC}}}\right]
\end{aligned}
$$

which we finally averaged over the tether length for use in simple estimates to follow.

However, not only is the lateral load on the tether at capture high but also the gravity gradient at Jupiter is weak. Kepler's law

$$
\Omega^{2}=\mu / a^{3} \propto \rho R^{3} / a^{3}
$$

shows that, with $\rho_{J}$ being one-fourth the Earth's density and other factors being equal, tether tension for a grazing $(a \approx R)$ Jovian orbit is one-fourth the tension in standard (roughly grazing) Low Earth Orbits. Furthermore, tether orbits along the Jovian mission are far from grazing; this makes the gravity gradient much weaker.
Setting the tether to spin with $\omega^{2} \gg 3 \Omega^{2}$ can provide the tension required to keep lateral bowing small. The tension under tether spin is obtained from (60) itself through the simple replacement $3 \Omega^{2} \Rightarrow \omega^{2}$, neglecting the gravity-gradient contribution

$$
F_{T} \approx \frac{\omega^{2} L^{2} \rho_{t} h w}{4}\left[\frac{M_{\mathrm{SC}}}{m_{t}}-\frac{2}{3}\right] .
$$

For a mass ratio $M_{\mathrm{SC}} / m_{t}=3.25$ and an aluminum foil of thickness $h=0.05 \mathrm{~mm}$ and $L=50 \mathrm{~km}$, a 12 -min spin (which is 50 times faster than $\Omega$ for a circular orbit at $r=a_{s}$ ) provides a tether tension

$$
F_{T} \approx 499 \mathrm{~N} \times w / 3 \mathrm{~cm} .
$$

This is over 1.5 times greater than $F_{L}$ as given in (58), thus satisfying condition (55). Note that the $F_{T} / F_{L}$ ratio is independent of width.

A high spin, however, may result in an unacceptable tensile stress $\sigma_{T}$, which reads

$$
\sigma_{T}=\frac{F_{T}}{w h}=\frac{\rho_{t} \omega^{2} L^{2}}{4}\left(\frac{M_{\mathrm{SC}}}{m_{t}}-\frac{2}{3}\right) .
$$

For $M_{\mathrm{SC}}=3.25 m_{t}$ and a $50-\mathrm{km}$ aluminum foil at a $12-\mathrm{min}$ spin, (63) yields

$$
\sigma_{T}=3.48 \times 10^{8} \frac{\mathrm{N}}{\mathrm{m}^{2}} .
$$

The tensile strength of some aluminum alloys can be twice as high. Alloys would have lower electrical conductivity than pure aluminum but one might still be in the no-ohmic effect limit, which is independent of conductivity.

Tether temperature may be an issue which further reduces the tensile strength. A possible alternative would be the use of a foil made of aluminized PBO (i.e., Zylon). The PBO fibers, with a tensile strength of $5.8 \mathrm{GN} / \mathrm{m}^{2}$, would carry the bulk of the tensile load and reinforce the tape against tearing while the aluminum would carry the current.

\section{CONCLUSION}

We have studied whether an ED tether can capture an SC with full mass $M_{\mathrm{SC}}$ a few times its mass $m_{t}$, in transfer from Earth, into a highly elliptical Jovian orbit. The (bare) tether would be a reinforced aluminum foil with tens of kilometer length $L$ and a fraction of millimeter thickness $h$, which collects electrons as an efficient Langmuir probe and can tap Jupiter's rotational energy for propulsion (and power). The capture operation is analyzed in this paper, capture being doubly critical for tethers because orbits, once closed, can substantially evolve under repeated propellantless (Lorentz) force. Design parameters $L$ and $h$ and capture perijove radius $r_{p}$ faced opposite criteria. Efficient capture (high $M_{\mathrm{SC}} / m_{t}$ ratio) requires a low $r_{p}$ and a high $L^{3 / 2} / h$ ratio. However, combined bounds on tether tensile stress, arising from a spin made necessary by the low Jovian gravity gradient, and tether bowing require a high $r_{p}$ and a low 
$L^{5 / 2} / h$ ratio. Bounds on tether temperature again require a high $r_{p}$ and a low $L^{3 / 8} /(\text { tether emissivity })^{1 / 4}$ ratio.

We found that an aluminum foil that is $50 \mathrm{~km}$ long and $0.05 \mathrm{~mm}$ thick would capture an SC that is 6.25 times as massive into a barely elliptical orbit with perijove at $1.3 R_{J}$; half that mass could be captured into an orbit of eccentricity of about 0.98 . The foil could be made of aluminized PBO (Zylon) coated to reach thermal emissivity of about 0.8 , to resist tearing and keep a high tensile strength at temperatures of a few hundred centigrade degrees. Ohmic effects are very weak throughout capture, allowing for a cross section that is not fully conductive.

Capture performance and constraints are independent of foil width $w$ and allow scaling over a broad mass range. Because of the low density and high temperature of electrons in the Jovian plasmasphere, the Debye length would be typically $1 \mathrm{~m}$ or larger. This results in a collection well within the OML regime for any reasonable tape width, as far as space charge effects are concerned. Maximum width would arise from requiring that $w$ be small against four times the electron thermal gyroradius throughout capture or small against $40 \mathrm{~cm}$, for example. In turn, a minimum value of width might arise from microparticle impact considerations.

With the Debye length much larger than $1 / 4 w$, a possible tape twist would not affect current collection [31]. Also, the actual magnetic-dipole tilt of Jupiter will have a negligible direct effect on tether capture. As shown in (7) and (8), both mechanical power and motional field component along the tether, if kept in the orbital plane, involve the magnetic field component perpendicular to that plane; for an equatorial orbit, the Jovian tilt of about $10^{\circ}$ reduces that component by roughly 1.5 percent. However, the small out-of-plane component of the Lorentz force might result in some out-of-plane instability. This will be discussed, along with the Lorentz torque on the tether, in a companion paper about a Jovian moon tour, also involving radiation-dose accumulation and power generation issues [28].

\section{REFERENCES}

[1] K. P. Klaasen, Status of the Europa Orbiter Mission, Europa Focus Group, Ames Res. Center, Feb. 1-2, 2001. [Online]. Available: hdl. handle.net/2014/16588

[2] R. Greeley and T. Johnson, "The Jupiter icy moons orbiter project: The scientific rationale," EOS, Trans. Amer Geophys. Union, vol. 85, no. 36, pp. 337-343, Sep. 2004.

[3] R. D. Abelson, "Europa Geophysical Explorer (EGE) mission concept," presented at the Space Technologies and Applications Int. Forum, Albuquerque, NM, Feb. 12-16, 2006. [Online]. Available: hdl.handle. net/2014/38764

[4] J. R. Spencer et al., "The future of Io exploration," in The Future of Solar System Exploration, 2003-2013, ser. Astronomical Soc. Pac. Conf., vol. 272, M. V. Sykes, Ed., 2002, pp. 201-216.

[5] J. F. Cooper, E. C. Sittler, Jr., S. J. Sturner, and J. Pitman, "Ganymede exploration orbiter-Jovian system observer," presented at the Outer Planets Assessment Group Meeting, Pasadena, CA, May 4-5, 2006. [Online]. Available: www.lpi.usra.edu/opag/may_06_meeting/ presentations/cooper_1

[6] P. Renard, C. Koeck, S. Kemble, A. Atzei, and P. Falkner, "System concepts and enabling technologies for an ESA low-cost mission to Jupiter/Europa," presented at the 55th Int. Astronautical Congr., Vancouver, BC, Canada, Oct. 4-8, 2004, Paper-04-Q.2a.02.

[7] S. C. Bolton, "Juno status report," presented at the Outer Planets Assessment Group Meeting, Arlington, VA, Oct. 6-7, 2005. [Online]. Available www.lpi.usra.edu/opag/oct_05_meeting/juno_status

[8] R. Grammier, "An overview of the Juno mission to Jupiter," in Proc. Int. Symp. Space Technol. Sci., Kanazawa, Japan, Jun. 4-11, 2006, pp. $1567-1575$.
[9] R. L. McNutt, Jr. et al., "Radioisotope electric propulsion: Enabling the decadal survey science goals for primitive bodies," presented at the Space Technologies and Applications Int. Forum, Albuquerque, NM, Feb. 12-16, 2006. [Online]. Available: www.lpi.usra.edu/opag/ menuttstaif06

[10] T. V. Johnson, K. Clark, R. Greeley, and R. Pappalardo, "Europa exploration: Challenges and solutions," presented at the 37th Lunar and Planetary Science Conf., League City, TX, Mar. 13-17, 2006. [Online]. Available: www.lpi.usra.edu/meetings/lpsc2006/pdf/1459

[11] K. B. Clark, R. T. Pappalardo, and R. Greeley, "Europa explorer: A mission to explore Europa and investigate its habitability," EOS Trans. AGU Fall Meeting Suppl., vol. 88, no. 52, 2007. Abstract P52A-08.

[12] J.-P. Lebreton, "Cosmic vision outer planets mission (OPM). TSSM: Titan/Saturn system mission. EJSM: Europa/Jupiter system mission," presented at the Outer Planets Assessment Group Meeting, Boulder, CO, Mar. 31-Apr. 1, 2008. [Online]. Available: www.lpi.usra.edu/opag/ march_08_meeting/presentation/lebreton

[13] P. A. Penzo, "A survey of tether applications to planetary exploration," Adv. Astronaut. Sci., vol. 62, pp. 71-88, 1986.

[14] W. E. Hammond, S. E. Freeman, M. J. Nave, and C. C. Rupp, "Jovian electrodynamic tether experiment," presented at the 29th Aerosp. Sci. Meeting, Reno, NV, Jan. 7-10, 1991, AIAA-91-0426 Paper.

[15] S. B. Gabriel, R. M. Jones, and H. B. Garrett, "Alfven propulsion at Jupiter," in Proc. 2nd Int. Conf. Tethers Space, Venice, Italy, Oct. 4-8, 1987, pp. 217-229.

[16] D. L. Gallagher, L. Johnson, J. Moore, and F. Bagenal, "Electrodynamic tether propulsion and power generation at Jupiter," NASA Marshall Space Flight Center, Huntsville, AL, NASA/TP-1998-208475, Jun. 1998.

[17] F. Bagenal, "Empirical model of the Io plasma torus: Voyager measurements," J. Geophys. Res., vol. 99, no. A6, pp. 11 043-11 062, Jun. 1994.

[18] M. Dobrowolny, Ed., "Special TSS-1 issue," Il Nuovo Cimento, vol. 17c, Jan./Feb. 1994.

[19] I. Katz et al., "Plasma turbulence enhanced current collection: Results from the plasma motor generator electrodynamic tether flight," J. Geophys. Res., vol. 100, no. A2, pp. 1687-1690, Feb. 1995.

[20] D. C. Thompson et al., "The current-voltage characteristics of a large probe in low Earth orbit: TSS-1R results," Geophys. Res. Lett., vol. 25 , no. 4, pp. 413-416, 1998.

[21] B. E. Gilchrist et al., "Enhanced electrodynamic tether currents due to electron emission from a neutral gas discharge: Results from the TSS-1R mission," Geophys. Res. Lett., vol. 25, no. 4, pp. 437-440, 1998.

[22] G. Vannaroni et al., "Current-voltage characteristic of the TSS-1R satellite: Comparison with isotropic and anisotropic models," Geophys. Res. Lett., vol. 25, no. 5, pp. 749-752, 1998.

[23] D. L. Cooke and I. Katz, "TSS-1R electron currents: Magnetic limited collection from a heated presheath," Geophys. Res. Lett., vol. 25, no. 5, pp. $753-756,1998$.

[24] L. Johnson, R. D. Estes, E. Lorenzini, M. Martinez-Sanchez, and J. R. Sanmartin, "Propulsive small expendable deployer system experiment," J. Spacecr. Rockets, vol. 37, no. 2, pp. 173-176, Mar./Apr. 2000.

[25] J. R. Sanmartin and E. C. Lorenzini, "Exploration of outer planets using tethers for power and propulsion," J. Propuls. Power, vol. 21, no. 3, pp. 573-576, May/Jun. 2005.

[26] J. R. Sanmartin, M. Martinez-Sanchez, and E. Ahedo, "Bare wire anodes for electrodynamic tethers," J. Propuls. Power, vol. 9, no. 3, pp. 353-360, Jun. 1993.

[27] J. G. Laframboise and L. W. Parker, "Probe design for orbit-limited current collection," Phys. Fluids, vol. 16, no. 5, pp. 629-636, May 1973.

[28] J. R. Sanmartin, M. Charro, E. C. Lorenzini, H. B. Garrett, C. Bombardelli, and C. Bramanti, "Electrodynamic tether at Jupiter-II: Fast moon tour after capture," IEEE Trans. Plasma Sci. submitted for publication.

[29] N. Divine and H. B. Garrett"Charged particle distributions in Jupiter's magnetosphere," J. Geophys. Res., vol. 88, no. A9, pp. 6889-6903, Sep. 1983.

[30] E. Ahedo and J. R. Sanmartin, "Analysis of bare-tether systems for deorbiting low-earth-orbit satellites," J. Spacecr. Rockets, vol. 39, no. 2, pp. 198-205, 2002. The tether-thrust efficiency, which equals $i_{a v}$, was wrongly approximated as $3 \hat{L}^{3 / 2}$

[31] B. E. Gilchrist, S. G. Bilen, E. Choiniere, A. D. Gallimore, and T. B. Smith, "Analysis of chamber simulations of long collecting probes in high-speed dense plasmas," IEEE Trans. Plasma Sci., vol. 30, no. 5, pp. 2023-2034, Oct. 2002. 